American Journal of Infectious Diseases 4 (1): 59-67, 2008

ISSN 1553-6203

(C) 2008 Science Publications

\title{
Anti-prM Antibody as an Autoantibody in Dengue Virus Infection
}

\author{
${ }^{1}$ Kao-Jean Huang, ${ }^{1}$ Yu-Tien Cheng, ${ }^{1}$ Yee-Shin Lin, ${ }^{4}$ Jyh-Hsiung Huang, ${ }^{1}$ Hsiao-Sheng Liu, \\ ${ }^{2}$ Trai-Ming Yeh, ${ }^{3}$ Ching-Chuan Liu and ${ }^{1}$ Huan-Yao Lei \\ ${ }^{1}$ Departments of Microbiology and Immunology \\ ${ }^{2}$ Department of Medical Technology \\ ${ }^{3}$ Department of Pediatrics, College of Medicine, National Cheng Kung University, Tainan \\ ${ }^{4}$ Center for Disease Control, Department of Health, Taipei, Taiwan, Republic of China
}

\begin{abstract}
We have reported that anti-prM antibody is an enhancing antibody that enhances DV infection of non-Fc receptor bearing cells by dual specificity. We identified the epitope recognized by this anti-prM antibody, M3, which is located at the a.a.53-67of the prM protein nearby the prM/M cleavage junction and these anti-M3 antibodies could be detected in dengue patients sera. Surprisingly, this anti-prM antibody not only recognizes the prM protein of dengue virions, but also cross-reacts with epithelial cells, endothelial cells as well as $\mathrm{T}$ cells. The binding of anti-prM antibody to endothelial cells was dose-dependent and could be blocked by M3 peptides. Human M3-specific antibodies from dengue patient sera were demonstrated to be able to bind to endothelial cells and mediate ADE infection on K562 cells. Furthermore, the anti-prM antibody will mediate the antibody dependent cell phagocytosis, leading to the similar severe form of DHF/DSS. In conclusion, anti-prM antibody plays two roles in the pathogenesis of dengue virus infection: to be an enhancing antibody and an autoantibody as well.
\end{abstract}

Key words: Autoimmunity, molecular mimicry, antibody, virology, endothelial cell

\section{INTRODUCTION}

According to WHO estimation, over 2.5 billion people living in tropical and subtropical areas were affected by dengue viruses and up to 50 million infections were reported annually ${ }^{[1]}$. There are four dengue virus serotypes transmitted by the main mosquito vector, Aedes aegypti, in the world ${ }^{[2]}$. Dengue virus infection causes disease spectrum from mild dengue fever to severe forms as dengue hemorrhagic fever and dengue shock syndrome (DHF/DSS $)^{[3]}$. The pathogenesis causing DHF/DSS remains elusive ${ }^{[4]}$. Antibody dependent enhancement theory was proposed to explain the increased viral load due to increase viral entry of monocytes through the antibody-virus complexes and $\mathrm{Fc}$ receptor pathway ${ }^{[5]}$. Thus, overt immune responses mounted by excess viral antigens lead to platelet dysfunction and reticulum endothelial cell damage, which were the two hallmarks of DHF/DSS ${ }^{[6]}$. We proposed an immunopathogenesis for DHF/DSS where autoimmunity may involve during dengue virus infection ${ }^{[7]}$. Anti-platelet and anti-endothelial cell antibodies could be detected in dengue patient sera and may consist of a portion of anti-dengue virus non-structure protein 1 (NS1) antibodies $^{[8,9]}$. Monoclonal anti-NS1 antibodies were proven to cross-react with integrin/adhesin proteins on platelets and endothelial cells ${ }^{[10]}$. Molecular mimicry defines that there are sequence or structural similarities between peptides from pathogens and self antigen ${ }^{[11]}$ and might play an active role in the generation of antiplatelet and anti-endothelial cell autoantibodies during dengue virus infection.

There are two forms of dengue virions in its life cycle, immature and mature particles ${ }^{[12]}$. During maturation process, immature viral particles turn out to be mature, accompanied with morphological change from spike-like protrusion to become smoother surface and 100 fold increase in infection activity ${ }^{[13]}$. Immature viral particles on their surface consist of (i) envelope glycoprotein (E) which is responsible for virus attachment and penetration and (ii) premembrane protein (prM) that was thought to protect virus pre-mature in virus maturation process during Golgi vesicle transportation. Antibodies against dengue $\mathrm{E}$ proteins were studied extensively, but little is known about anti-prM antibody. Monoclonal anti-prM antibodies have been reported to mediate ADE

Corresponding Author: Huan-Yao Lei, PhD, Department of Microbiology and Immunology, College of Medicine, National Cheng Kung University, Tainan, Taiwan, Republic of China 
infection and provide cross-protection against four dengue virus serotypes in vivo ${ }^{[14]}$.

As there are huge amounts of auto-reactive antibodies during dengue virus infection, we extend our previous finding that in addition to mediate ADE infection anti-prM antibody may bind to BHK and A549 cells ${ }^{[15]}$. We then examined if this anti-prM antibody will cross-react with other cell types, such as endothelial cells and may play a role as an autoantibody during dengue virus infection.

\section{MAETERIALS AND METHODS}

Cells, viruses and reagents: Cells including C6/36, K562, THP-1, Jurkat, BHK and A549 were maintained in Dulbeccos Modified Eagle (DME) medium supplemented with $10 \%$ fetal bovine serum (FBS). Dengue virus serotype 2 strain (PL046) was obtained from Center of Disease Control (CDC) in Taiwan and was propagated in C6/36 mosquito cells. The harvested virus titer was determined by standard plaque assay on BHK cells as previously described ${ }^{[16]}$. The human dengue virus serotype 3 immune sera were obtained from the outbreak in Taiwan in 1998 and the control sera were collected from healthy donors with no history of dengue virus infection. Monoclonal anti-dengue virus E (50-2, 185-10), anti-prM (70-21) and anti-NS1 (206-35) antibodies were purified from hybridoma culture supernatant using Montage Prosep-G kit (Millipore, Bedford, USA). Biotinylated anti-dengue virus NS1 monoclonal antibodies were obtained by using NHS-LC-Biotin labeling kit (Pierce, Rockford, USA). Control mouse IgG1, IgG2a and streptavidinFITC were obtained from eBioscience (eBioscience, San Diego, CA) and FITC-, TRITC-, peroxidaseconjugated goat anti-mouse IgG or goat anti-human IgG secondary antibodies were purchase from Jackson Laboratories (Jackson ImmunoResearch Laboratories, West Grove, USA).

Immunofluorescence staining: BHK cells were cultured in the Lab-Tek II chamber slide (Nunc, Roskilde, Denmark) and were infected by dengue virus at the multiply of infection of $5(\mathrm{MOI}=5)$. After $24 \mathrm{~h}$ incubation, the cells were fixed with cold methanol for $10 \mathrm{~min}$ and then stained with control immunoglobulins, or purified dengue monoclonal antibodies for $1 \mathrm{~h}$ at $4^{\circ} \mathrm{C}$, followed by incubation with FITC- or TRITCcojugated goat anti-mouse $\mathrm{IgG}$ for another $1 \mathrm{~h}$ at $4^{\circ} \mathrm{C}$. Finally, the cells were counterstained with Hoechst 33258 (Sigma-Aldrich Co, St. Louis, USA) at $5 \mu \mathrm{g} \mathrm{mL} \mathrm{m}^{-1}$ for $15 \mathrm{~min}$ and then mounted with Vectashield H-1000 (Vector Laboratories, Burlingame,
USA) mounting medium. The fluorescence image were captured and processed by fluorescence microscope (Leica Geosystems AG, St. Gallen, Switzerland).

Immunoprecipitation of dengue virions by antidengue monoclonal antibody: Purified dengue monoclonal antibodies were coated on magnetic Dynabeads M-450 GAM IgG (Dynal Biotech, Oslo, Norway) according to the manufacture's instruction. Dengue virus-infected C6/36 cell culture supernatant was incubated with individual control Ig, dengue anti-E (185-10) or anti-prM (70-21) monoclonal antibody-coated Dynabeads for $1 \mathrm{~h}$ at $4{ }^{\circ} \mathrm{C}$ with gentle agitation. After several washing with $1 \%$ Triton-X $100 / \mathrm{PBS}$, the immune-complexes were eluted by $0.1 \mathrm{M}$ glycine/HCL, $\mathrm{pH} 2.5$ and then neutralized by $1 \mathrm{M}$ Tris, $\mathrm{pH} 9.0$ with the amount of $1 / 10$ volume of elution buffer. The eluted proteins were subjected to Western blot analysis by using anti-E (50-2) or anti-prM (70-21) monoclonal antibody followed by staining with secondary peroxidase-conjugated goat anti-mouse IgG antibody. By applying the Western Lightning Chemiluminescence Ragent Plus (Amersham Bioscience Ltd, Piscataway, USA), the reactive proteins were visualized on exposed film.

Peptide enzyme-link immunosorbent assay (ELISA): The 96-well microtiter plate was coated overnight at $4^{\circ} \mathrm{C}$ with individual synthetic peptides (NS1-P1, M1 to M6) at concentration of $10 \mu \mathrm{g} \mathrm{mL}^{-1}, 100 \mu \mathrm{L}$ per well in coating buffer $\left(0.1 \quad \mathrm{M} \mathrm{Na}_{2} \mathrm{CO}_{3} / \mathrm{NaHCO}_{3}, \mathrm{pH}\right.$ $9.5)$ as previously described ${ }^{[17]}$. After washing with PBS- $0.1 \%$ Tween 20 (PBST), wells were blocked with $200 \mu \mathrm{L}$ of PBS-1\% bovine serum albumin (BSA) for 1 $\mathrm{h}$ at $37^{\circ} \mathrm{C}$ and then incubated for $1 \mathrm{~h}$ at $37^{\circ} \mathrm{C}$ with 100 $\mu \mathrm{L}$ of $10 \mu \mathrm{g} \mathrm{mL}^{-1}$ monoclonal antibodies or $100 \mu \mathrm{L}$ of 1:100 diluted sera in PBST-1\% BSA. A 1:5,000 dilution of horse radish peroxides-conjugated goat antimouse IgG (for mouse monoclonal antibodies) or antihuman IgG (for clinical patient serum) were incubated for another $1 \mathrm{~h}$ at $37^{\circ} \mathrm{C}$. Finally, the color was developed by the addition of the chromogen, Tetramethylbenzidine (TMB, Clinical Science= products, Mansfield, USA) and the enzyme activities were terminated by the addition of $2 \mathrm{~N} \mathrm{H}_{2} \mathrm{SO}_{4}$. The absorbance at $450 \mathrm{~nm}$ was measured with the Emax microplate reader (Molecular Devices Corporation, Sunnyvale, USA).

Flow cytometric assay for binding of anti-prM mAb to various cell lines: The adherent cells, such as BHK, A549, HUVEC and HMEC-1, were detached by $5 \mathrm{mM}$ EDTA/PBS and re-suspended as single cell suspension 
like Jurkat cell suspension before staining with indicative amounts of control, anti-prM (70-21), anti-E (185-10) or anti-NS1(206-35) mAbs. After several wash, these cells were stained with FITClabeled goat anti-mouse IgG secondary antibody. Binding fluorescence intensity was determined by flow cytometry. For isolated human antibodies or diluted patient serum, FITC-labeled goat anti-human IgG secondary antibody was used. For the M3 peptide blocking experiment, anti-prM antibodies $(2 \mu \mathrm{g})$ were pre-incubated with various amounts of M3 peptides $(0-20 \mu \mathrm{g})$ or control peptide, M1 peptide $(10 \mu \mathrm{g})$, before incubation with HMEC-1 cells.

Isolation of peptide-specific antibodies: M3 peptides were immobilized onto the magnetic beads Epoxy 270 (Dynal Biotech, Oslo, Norway) according the manufacturer's user guide. The dengue immune serum from the outbreak in Taiwan in 1998 was incubated with M3 peptide-magnetic beads for $1 \mathrm{~h}$ at $4{ }^{\circ} \mathrm{C}$ with gentle agitation. and the M3 peptide-reactive human immunoglobulins were eluted from magnetically separated-beads by using $0.1 \mathrm{M}$ glycine/HCL pH2.5. This eluted antibody solution was immediately neutralized by adding $1 / 10$ the volume of elution buffer with $1 \mathrm{M}$ Tris-HCl, $\mathrm{pH} 9.0$ and its concentration was determined by measuring the absorbance at $280 \mathrm{~nm}$ wave-length.

Flow cytometric detection of dengue virus-infected cells: The virus-antibody complexes were prepared by mixing diluted M3-specific human antibodies with viruses $(\mathrm{MOI}=5)$ for $30 \mathrm{~min}$ at $37^{\circ} \mathrm{C}$ before addition to the K562 cell suspension ( $2 \times 10^{5} /$ sample). Infection was performed at $37^{\circ} \mathrm{C}$ for $90 \mathrm{~min}$. After virus adsorption, cells were washed twice and resuspended in complete medium. At $24 \mathrm{hrs}$ time point, these cells were harvested and fixed by $2 \%$ paraformaldehyde in PBS for $20 \mathrm{~min}$ on ice. After fixation, they were washed with PBS twice and then stained with appropriate amounts of biotinylated anti-dengue NS1 (206-35) monoclonal antibodies in the permeabilization buffer ( $2 \% \mathrm{FBS}, 0.1 \%$ saponin, $0.1 \%$ sodium azide in PBS) for $30 \mathrm{~min}$ on ice. These cells were washed with permeabilization buffer twice and then stained with streptavidin-FITC in the same buffer for another $30 \mathrm{~min}$. Finally, these cells were washed again with the permeabilization buffer and resuspended in staining buffer (2\% FBS, $0.1 \%$ sodium azide in PBS) for flow cytometric analysis by FACSCalibur (Becton Dickinson Immunocytometry Systems, San Jose, CA). Flow-cytometric data were re-analyzed using the program, WinMDI version 2.8.
Flow cytometric detection of antibody-dependent cell phagocytosis: Jurkat cells $\left(5 \times 10^{5}\right)$ were labeled with Green fluorescence dye, PKH67 (Sigma-Aldrich co, St. Louis, USA) and then coated with anti-prM (70-21), anti-E (185-10) or anti-NS1 (206-35) monoclonal antibodies $(5 \mu \mathrm{g})$. These labeled Jurkat cells were then incubated with either resting or preactivated THP-1 monocytes $\left(2 \times 10^{5}\right)$, which were activated by PMA $\left(10 \mathrm{ng} \mathrm{mL}^{-1}\right)$ plus IFN- $\gamma(30$ IU mL $\left.{ }^{-1}\right) 24 \mathrm{~h}$ earlier in 24-wells plate. After $2 \mathrm{~h}$ coincubation, cells were harvested and stained with PEconjugated anti-human CD14 antibody. All samples were analyzed by flow cytometer. The percentage of phagocytosis was defined as double positive cells (green and red fluorescence)/(cells with single red fluorescence+double positive cells)x $100 \%$. For dengue patient sera, non-dengue serum or DV3 immune sera (1:25) were used instead of anti-dengue virus monoclonal antibodies. Fluorescence beads, FluoresbriteTM Carboxylate 0.75 Micron Microspheres (Polyscience Inc, Warrington, USA), were used to evaluate the basal level of phagocytic activity of activated THP-1 monocytes.

\section{RESULTS}

Dengue virions were immunoprecipitated by monoclonal anti-prM antibody: In previous study, anti-dengue $\mathrm{E}$ antibodies were known to be able to enhance or neutralize dengue virus infection, but little is known about the anti-prM antibody. In our study, monoclonal anti-prM antibody may mediate the ADE infection of cells either with or without Fc receptors. This anti-prM antibody could also mediate ADE infection of all four dengue serotypes. It specifically recognized prM protein in immunofluorescence staining (Fig. 1A) and western blot analysis (data not shown) and could be used for immunoprecipitating dengue virions from dengue virus-infected C6/36 cell culture supernatant (Fig. 1B).

Monoclonal anti-prM antibodies recognize M3 epitope: In order to know the epitope recognized by anti-prM antibody, synthetic peptides (M1 to M6, NS1-P1) derived from prM or NS1 proteins were coated on ELISA plate for screening of anti-prM antibody binding. Our anti-prM antibody specifically recognize M3 peptide which is located at the a.a.53-67of the prM protein nearby the $\mathrm{prM} / \mathrm{M}$ cleavage junction (Fig. 1C). In addition, patients sera from dengue outbreak in Taiwan in 1998 contain these M3-specific antibodies (Fig. 1D). 
Am. J. Infect. Dis., 4 (1): 59-67, 2008
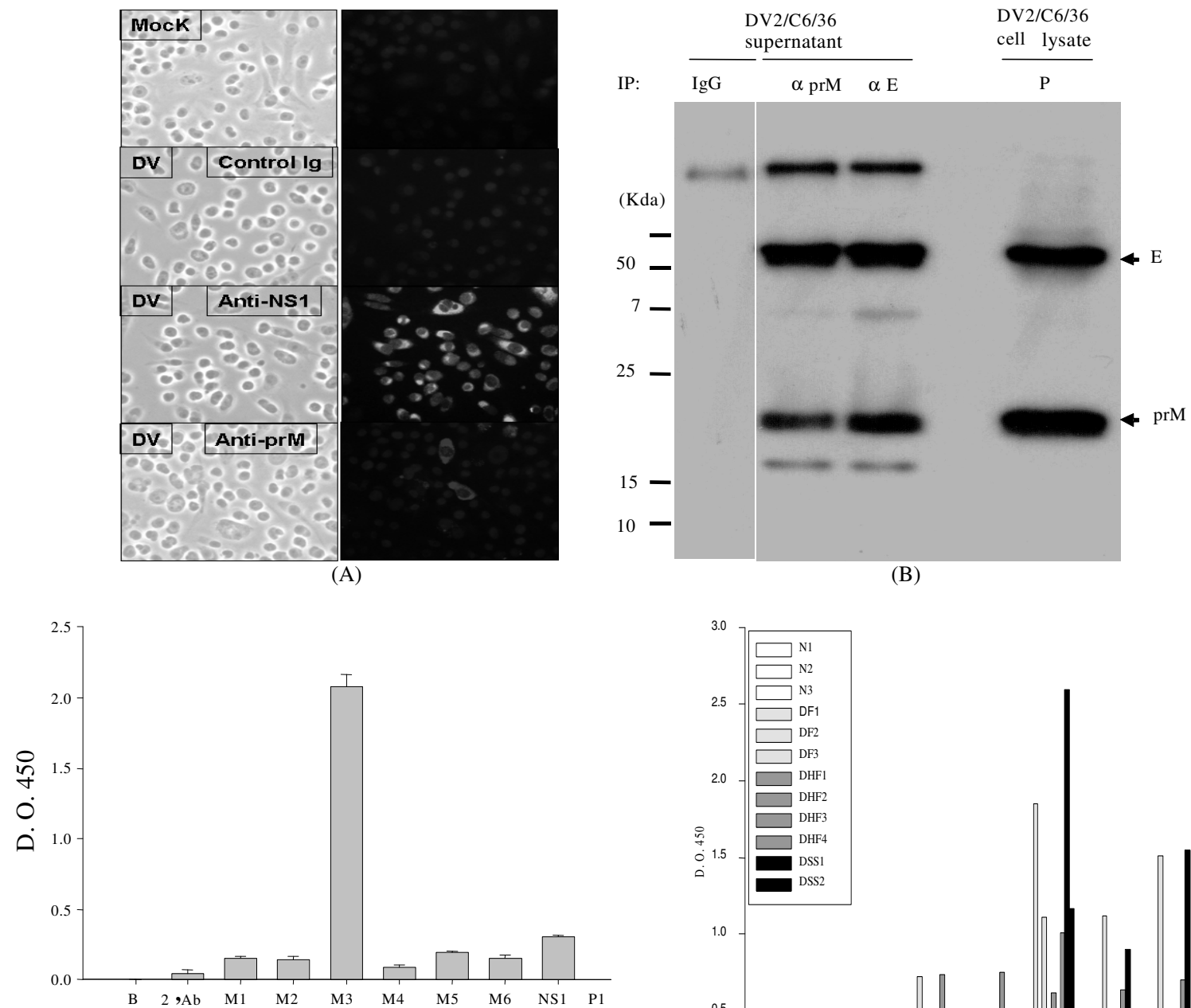

M1: h-mafhlttrngephmi-oh, M2: h-mivsrqekgksllfk-oh, M3: hcpflkqnepedidcw-oh, M4: h-gtctttgehrrekrsval-oh, M5: hvgmgletrtetwmss-oh, M6: h-wmssegawkhaqrie-oh, Ns1-p1: hdsgcvvswknkelkc-oh

(C)

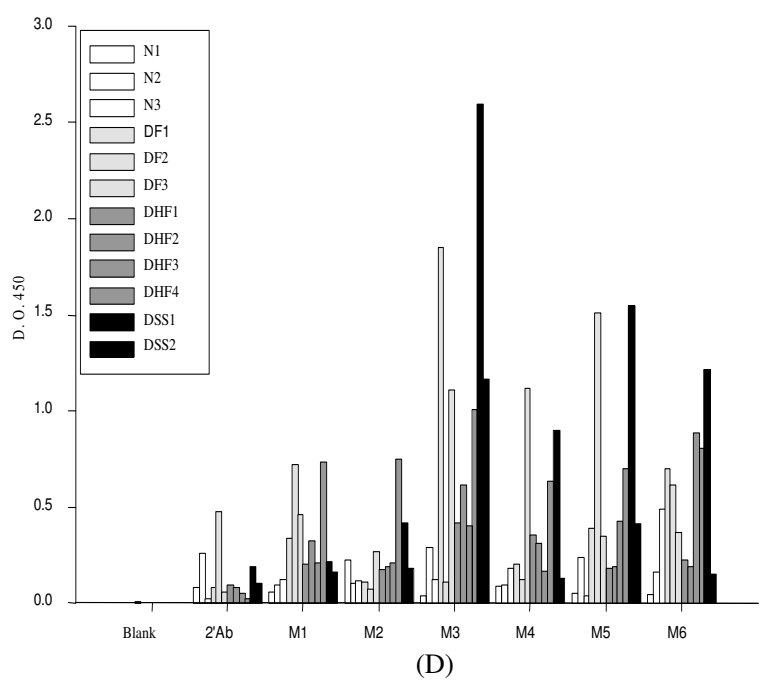

Fig. 1: Anti-prM antibody in dengue patients' sera. A: Immunofluorence staining of anti-prM Ab. B: Immunoprecipitation of dengue virions by anti-prM Ab. C: Epitope mapping of monoclonal anti-prM Ab. D: Human M3 peptide-specific antibodies in dengue patients's sera

Anti-prM antibodies cross-react with endothelial cells and lymphocytes: In our study, we found that anti-prM antibody may recognize A549 (lung epithelial cell) and BHK (baby kidney cell) cells (Fig. 2A and B). We then test if this anti-prM antibody will recognize other kinds of cells. Anti-prM antibody will cross-react with endothelial cells as well as $\mathrm{T}$ lymphocytes, including human microvascular endothelial cell -1 (HMEC-1), human umbilical vein endothelial cell (HUVEC) and Jurkat T cells (Fig. 3A). This crossreaction of anti-prM antibody to endothelial cells was dose-dependent (Fig. 3B) and was dose-dependently inhibited by M3 peptides (Fig. 3C).

Isolated human anti-prM antibodies may mediate the ADE infection and endothelial cell binding: As M3-specific human antibodies were detected in dengue patients sera, we coated M3 peptides on magnetic beads to isolate those antibodies. Serum with the highest M3 titer was used and M3-specific antibodies were purified (Fig. 4A). Isolated human M3-specific antibodies, with the function, were able to enhance dengue virus 


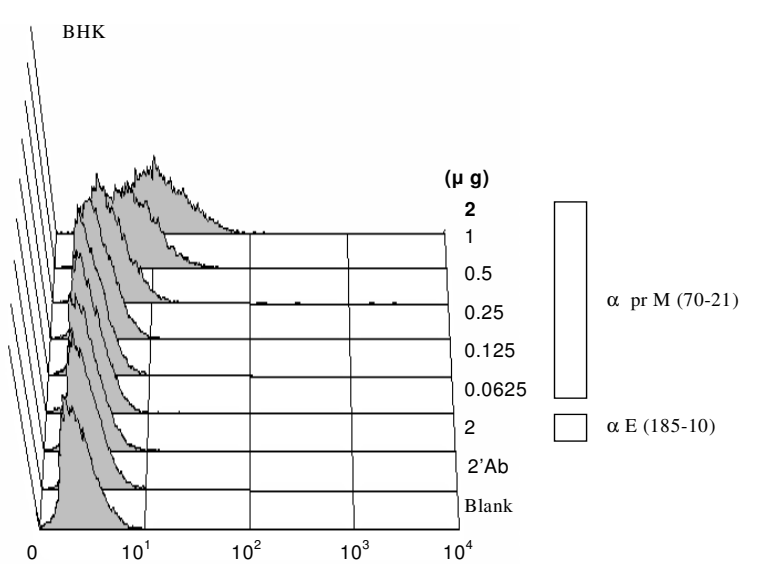

(A)

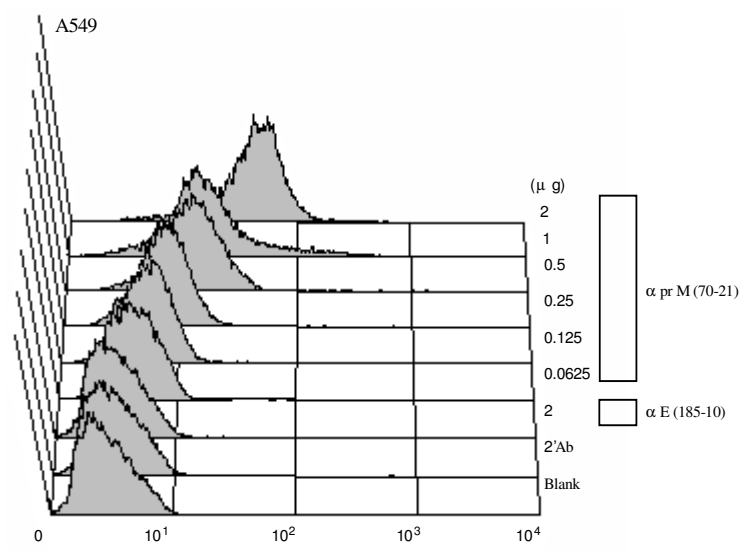

(B)

Fig. 2: Dose-dependent binding of anti-prM Ab to BHK (A) or A549 (B) cells

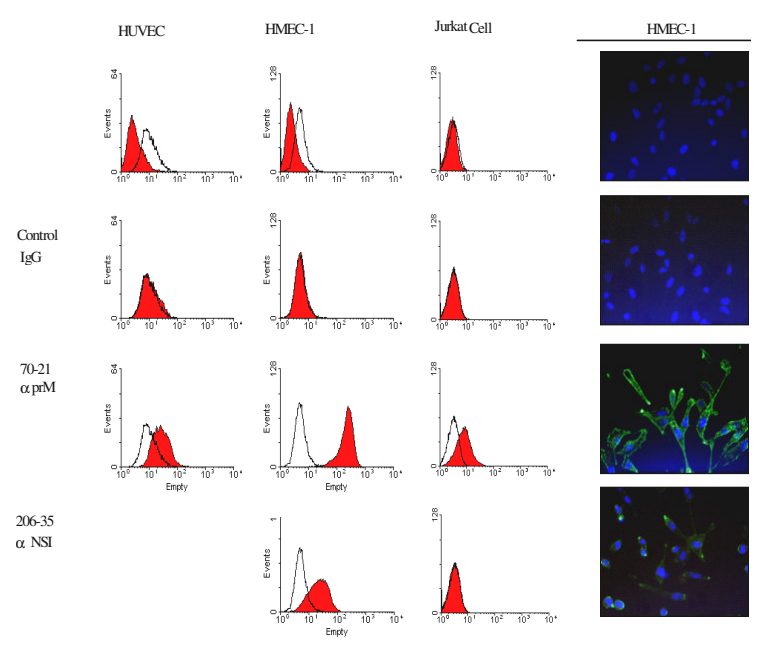

(A)

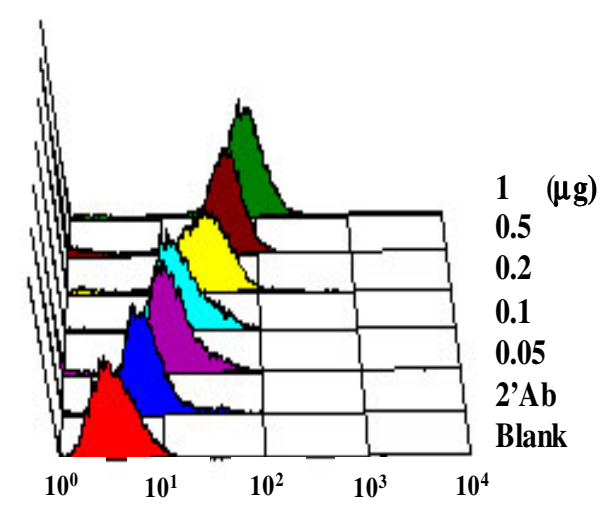

(B)

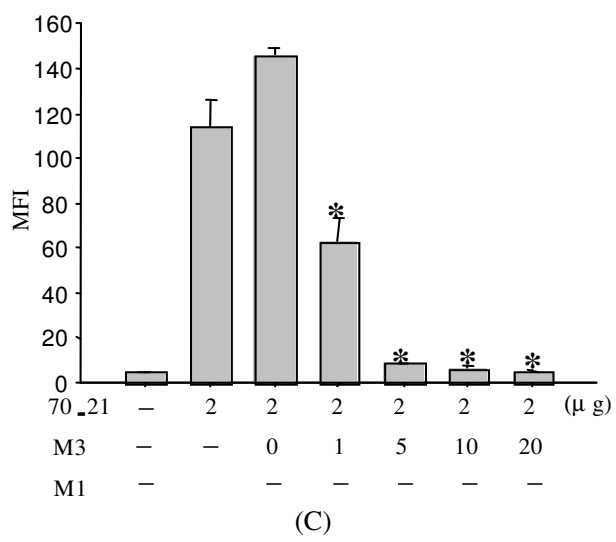

Fig. 3: Binding of anti-prM antibody to endothelial cells. (A) Binding of anti-prM antibody to endothelial cells or T cells. (B) Dose-dependent binding of anti-prM Ab to HMEC-1. (C) Blocking of anti-prM Ab binding to endothelial cells by M3 peptides 

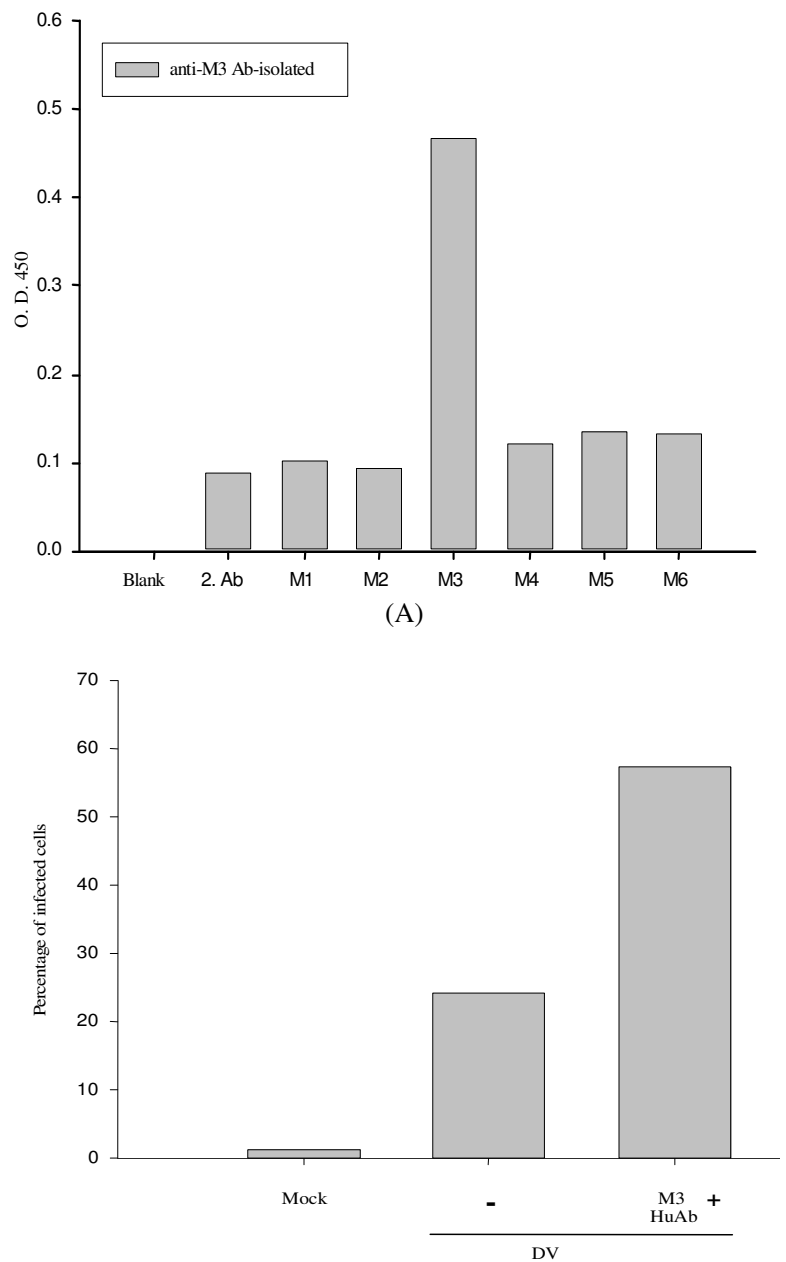

(B)
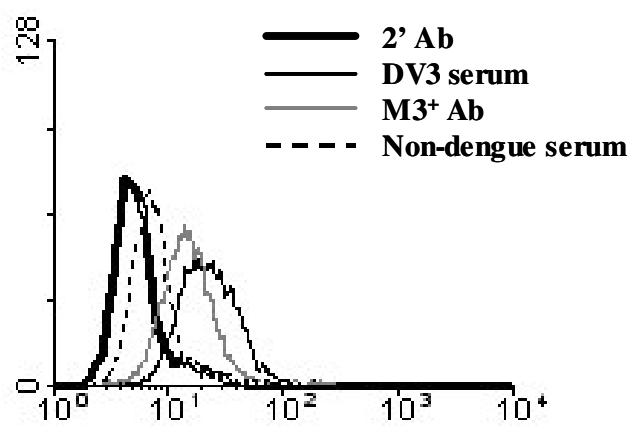

(C)

Fig. 4: Human anti-prM antibodies mediated ADE infection and endothelial binding. (A) Isolated $\mathrm{M} 3$-specific human $\mathrm{Ab}$ from dengue patient serum. (B) Human anti-M3 antibodies mediated the ADE infection on K562 cells. (C) Binding of human anti-M3 antibodies to endothelial cells
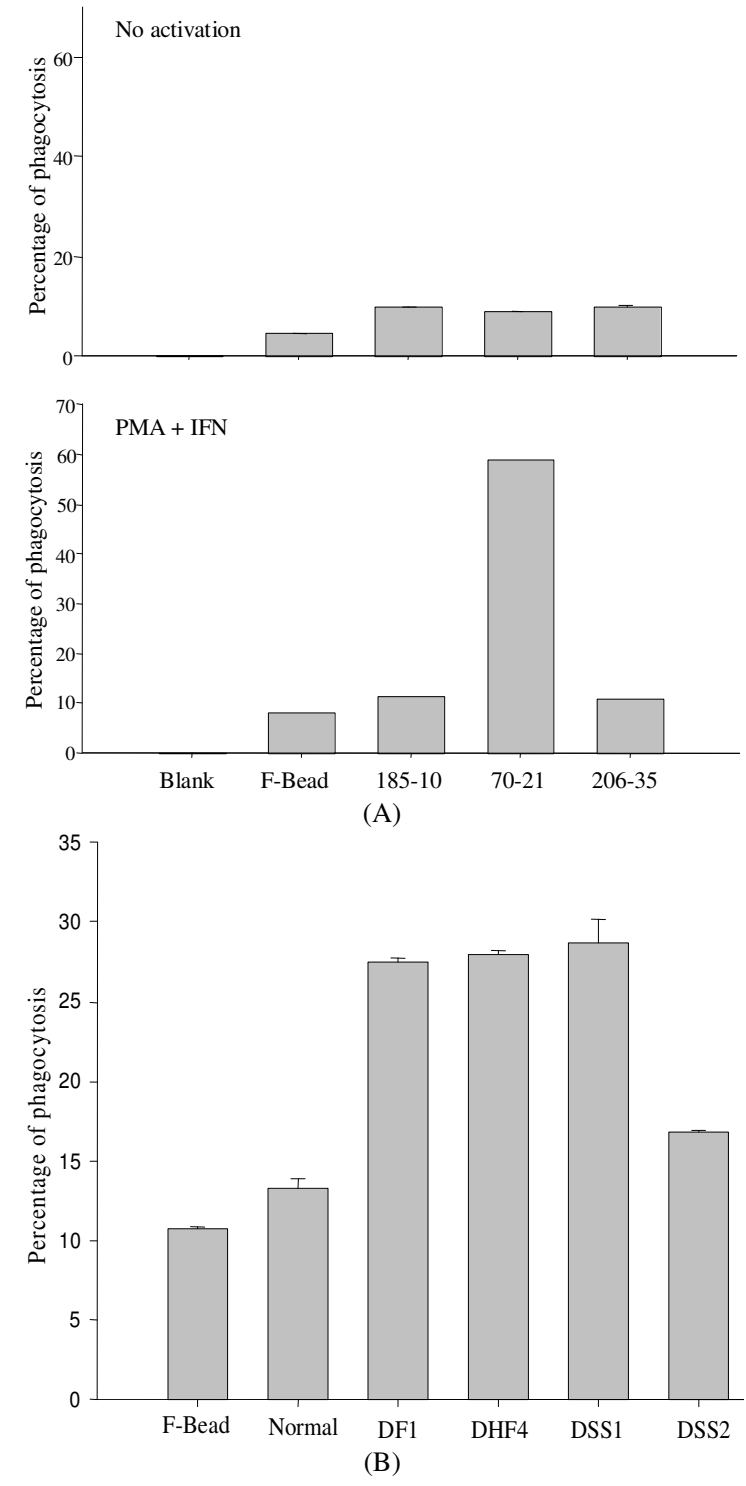

Fig. 5: Anti-prM antibody-mediated antibodydependent cell phagocytosis. (A) Monoclonal anti-prM $\mathrm{Ab}$ mediated Ab-dependent cell phagocytosis. (B) Dengue patient sera mediated Ab-dependent cell phagocytosis

infection (Fig. 4B) and to bind to endothelial cells (Fig. 4C).

Anti-prM antibody may mediate antibodydependent cell phagocytosis: As anti-prM antibody will cross-react with many kinds of cells, including epithelial cells, endothelial cells and lymphocytes. We test the hypothesis if anti-prM antibody could mediate the antibody-dependent cell phagocytosis. Human 
Jurkat T cells were coated with anti-NS1(206-35), antiprM (70-21) or anti-E (185-10) antibodies before incubating with resting or PMA/IFN- $\gamma$-activated THP-1 monocytes and only Jurkat $\mathrm{T}$ cell bound anti-prM antibody could enhance cell phagocytosis of activated monocytes (Fig. 5A). Incubation of $\mathrm{T}$ cells with inactivated-dengue patient sera (DF1, DHF4, DSS1 and DSS2) will also mediate the antibody-dependent cell phagocytosis (Fig. 5B).

\section{DISCUSSION}

The pathogenesis of DHF/DSS remains elusive. The ADE theory may explain the higher viral load in DHF/DSS as compared with DF. Then the increased viruses will trigger inadequate immune responses including cytokine overproduction, aberrant $\mathrm{T}$ cell response and complement activation, affecting the liver, the reticuloendothelial system and the vascular system to develop DHF/DSS. In dengue patients sera, more anti-platelet and anti-endothelial cell antibodies were detected in DHF/DSS than in DF and most of them were cross-reacting with dengue virus $\mathrm{NS}^{[8,9]}$. Especially, the endothelial cell cross-reactive anti-NS1 antibody will induce cell apoptosis through nitric oxide (NO) production ${ }^{[18]}$. Monoclonal anti-NS1 antibodies were reported to cross-react with thrombocytes, endothelial cells and human clotting factors, such as fibrinogen ${ }^{[10]}$. In addition, anti-E antibodies were also reported to bind to plasminogen and inhibit plasmin activity ${ }^{[19-21]}$. In this study, we demonstrated that except anti-NS1 and anti-E antibodies, anti-prM antibody could be an autoantibody and may cross-react with epithelial cells, kidney cells, endothelial cells and even $\mathrm{T}$ lymphocytes. M3 epitope recognized by monoclonal anti-prM antibody was equivalent to human, as dengue patient sera contain these M3 peptide-specific antibodies. Isolated human M3-specific human antibodies with the same ability to bind to endothelial cells and could mediate ADE infection. In the presence of activated mocrophages, autoantibody including antiprM antibody could mediate the antibody-dependent cell phagocytosis, which may happen in the liver and bone marrow as hemophagocytic syndrome-like pattern in DHF/DSS ${ }^{[22-24]}$. Thus, autoimmunity may participate in dengue virus infection and contribute to the pathogenesis of DHF/DSS.

Immune responses to prM proteins were studied in dengue patients' sera ${ }^{[25]}$ and in mouse model ${ }^{[26]}$. In clinical, antibodies against prM protein could be used as a diagnostic marker to distinguish previous infection of dengue virus from Japanese encephalitis virus ${ }^{[27]}$. The M3 epitope which is conserved among all four dengue virus serotypes but not other flavivirus, such as Japanese encephalitis virus, recognized by our own anti-prM antibody was located at the a.a.53-67of the prM protein nearby the $\mathrm{prM} / \mathrm{M}$ cleavage junction and was different from the published sequence recognized by other anti-prM monoclonal antibody $(2 \mathrm{H} 2)$, 40-PGFTVMAAIL-49 (M40-49) from the first membrane-spanning domain of the $\mathrm{M}$ protein ${ }^{[14]}$. Although our own anti-prM antibody may recognize all four dengue serotypes, this binding was insufficient to inhibit virus infection. The epitope recognized by our own monoclonal antibodies shares the same sequence of that B20-2 peptide derived from prM, which wont induce neutralizing antibodies in an animal study ${ }^{[26]}$. Taken together, we identified a novel epitope on prM protein, which may be useful for clinical diagnosis.

Molecular mimicry theory has been proposed for the production of auto-reactive antibodies ${ }^{[28]}$. In dengue patient sera, antibodies against platelets and endothelial cells were detected. In our findings, in addition to antiNS1 antibody, anti-prM antibody could also cross-react with either epithelial cell (A549) or endothelial cells (HMEC-1 and HUVEC). This binding was dosedependent and could be blocked by M3 peptides. One of the autoantigens recognized by anti-prM antibody on the surface of BHK cell or A549 cell was identified as heat shock protein 60 (HSP60), but the other autoantigens on endothelial cells or lymphocytes have not yet been identified. HSP60 have been reported as autoantigens in many diseases, including atherosclerosis ${ }^{[29]}$ and systemic lupus erythematosus ${ }^{[30]}$. Anti-Cytomegalovirus (CMV) viral proteins, UL122 and US28 may crossreact with HSP60 ${ }^{[31]}$. Anti-HSP60 antibody binding will directly cause endothelial cell apoptosis or indirectly damage those through complement-mediated cytotoxicity or antibodydependent cellular cytotoxicity in the presence of complement or human peripheral mononuclear cells ${ }^{[32]}$. Collectively, molecular mimicry mechanism seems to play an active role during dengue virus infection and the induction of such autoreactive antibodies may in part contribute to the pathogenesis of DHF/DSS.

Dengue vaccines have been developed for several decades ${ }^{[33-35]}$. The main problem of live-attenuated tetravalent vaccines or the subunit vaccines using the dengue viral structure proteins, such as E, prM or $\mathrm{E} / \mathrm{prM}$ proteins will encounter the ADE phenomena. If the induced-neutralizing titer decreases with time, it will put the vaccinated people at great risk to develop the severe symptoms when nature infection of dengue virus occurs. From the results of ours and others, vaccination with above vaccines may also induce the mimicry autoantibodies and this should be an issue for 
future vaccine design. It may be important to identify various kinds of epitopes, which are responsible for enhancing antibodies, neutralizing antibodies or autoreactive antibodies. On the other way, the autoantigens cross-reacted by anti-dengue virus antibodies should be identified, especially on thrombocytes or endothelial cells and whether the cells are directly or indirectly damaged by autoantibody engagement.

In conclusion, our findings support that anti-prM antibody could be not only an enhancing antibody to enhance dengue virus infection but also an autoantibody that may damage cells through either complement-mediated cytotoxicity or antibodydependent cell phagocytosis.

\section{ACKNOWLEDGMENT}

This work was supported by grant NSC95-3112B006-004 from National Science Council, Taiwan.

\section{COMPETING INTERESTS STATEMENT}

The authors declare that they have no competing financial interests.

\section{REFERENCES}

1. Gubler, D.J., 2002. The global emergence/resurgence of arboviral diseases as public health problems. Arch. Med. Res., 33: 330-342.

2. Henchal, E.A. and J.R. Putnak, 1990. The dengue viruses. Clin. Microbiol. Rev., 3: 376-396.

3. Sabin, A.B., 1952. Research on dengue during World War II. Am. J. Trop. Med. Hyg., 1: 30-50.

4. Green, S. and A. Rothman, 2006. Immunopathological mechanisms in dengue and dengue hemorrhagic fever. Curr. Opin. Infect. Dis., 19: 429-436.

5. Halstead, S.B., 1982. Immune enhancement of viral infection. Prog. Allergy, 31: 301-364.

6. Bhamarapravati, N., 1989. Hemostatic defects in dengue hemorrhagic fever. Rev. Infect. Dis., 11: S826-829.

7. Lei, H.Y., T.M. Yeh, H.S. Liu, Y.S. Lin, S.H. Chen and C.C. Liu, 2001. Immunopathogenesis of dengue virus infection. J. Biomed. Sci., 8: 377-388.

8. Lin, C.F., H.Y. Lei, C.C. Liu, H.S. Liu, T.M. Yeh, S.T. Wang, T.I. Yang, F.C. Sheu, C.F. Kuo and Y.S. Lin, 2001. Generation of IgM anti-platelet autoantibody in dengue patients. J. Med. Virol., 63: $143-149$.
9. Lin, C.F., H.Y. Lei, A.L. Shiau, C.C. Liu, H.S. Liu, T.M. Yeh, S.H. Chen and Y.S. Lin, 2003. Antibodies from dengue patient sera cross-react with endothelial cells and induce damage. J. Med. Virol., 69: 82-90.

10. Falconar, A.K., 1997. The dengue virus nonstructural-1 protein (NS1) generates antibodies to common epitopes on human blood clotting, integrin/adhesin proteins and binds to human endothelial cells: potential implications in haemorrhagic fever pathogenesis. Arch. Virol., 142: 897-916.

11. Fujinami, R.S. and M.B. Oldstone, 1985. Amino acid homology between the encephalitogenic site of myelin basic protein and virus: mechanism for autoimmunity. Science, 230: 1043-1045.

12. Zhang, Y., J. Corver, P.R. Chipman, W. Zhang, S.V. Pletnev, D. Sedlak, T.S. Baker, J.H. Strauss, R.J. Kuhn and M.G. Rossmann, 2003. Structures of immature flavivirus particles. Embo J., 22: 2604-2613.

13. Zhang, Y., W. Zhang, S. Ogata, D. Clements, J.H. Strauss, T.S. Baker, R.J. Kuhn and M.G. Rossmann, 2004. Conformational changes of the flavivirus E glycoprotein. Structure (Camb), 12: 1607-1618.

14. Falconar, A.K., 1999. Identification of an epitope on the dengue virus membrane $(\mathrm{M})$ protein defined by cross-protective monoclonal antibodies: design of an improved epitope sequence based on common determinants present in both envelope (E and M) proteins. Arch. Virol., 144: 2313-2330.

15. Huang, K.J., Y.C. Yang, Y.S. Lin, J.H. Huang, H.S. Liu, T.M. Yeh, S.H. Chen, C.C. Liu and H.Y. Lei, 2006. The dual-specific binding of dengue virus and target cells for the antibodydependent enhancement of dengue virus infection. J. Immunol., 176: 2825-2832.

16. Huang, K.J., S.Y. Li, S.C. Chen, H.S. Liu, Y.S. Lin, T.M. Yeh, C.C. Liu and H.Y. Lei, 2000. Manifestation of thrombocytopenia in dengue-2virus-infected mice. J. Gen. Virol., 81: 2177-2182.

17. Huang, J.H., J.J. Wey, H.F. Lee, T.L. Tsou, C.S. Wu, J.R. Wu, H.M. Chen, C. Chin, L.J. Chien, L.K. Chen, Y.C. Wu, M.J. Pan and T.M. Wang, 1996. Identification of immunodominant, groupspecific and subcomplex-specific, continuous epitopes in the core regions of Japanese encephalitis virus using synthetic peptides. Virus Res., 41: 43-53. 
18. Lin, C.F., H.Y. Lei, A.L. Shiau, H.S. Liu, T.M. Yeh, S.H. Chen, C.C. Liu, S.C. Chiu and Y.S. Lin, 2002. Endothelial cell apoptosis induced by antibodies against dengue virus nonstructural protein 1 via production of nitric oxide. $J$. Immunol., 169: 657-664.

19. Markoff, L.J., B.L. Innis, R. Houghton and L.S. Henchal, 1991. Development of cross-reactive antibodies to plasminogen during the immune response to dengue virus infection. J. Infect. Dis., 164: 294-301.

20. Chungue, E., L. Poli, C. Roche, P. Gestas, P. Glaziou and L.J. Markoff, 1994. Correlation between detection of plasminogen cross-reactive antibodies and hemorrhage in dengue virus infection. J. Infect. Dis., 170: 1304-1307.

21. Huang, Y.H., B.I. Chang, H.Y. Lei, H.S. Liu, C.C. Liu, H.L. $\mathrm{Wu}$ and T.M. Yeh, 1997. Antibodies against dengue virus $\mathrm{E}$ protein peptide bind to human plasminogen and inhibit plasmin activity. Clin. Exp. Immunol., 110: 35-40.

22. Wong, K.F., J.K. Chan, J.C. Chan, W.W. Lim and W.K. Wong, 1991. Dengue virus infectionassociated hemophagocytic syndrome. Am. J. Hematol., 38: 339-340.

23. Rueda, E., A. Mendez and G. Gonzalez, 2002. [Hemophagocytic syndrome associated with dengue hemorrhagic fever]. Biomedica., 22: 160-166.

24. Veerakul, G., K. Sanpakit, V.S. Tanphaichitr, C. Mahasandana and N. Jirarattanasopa, 2002. Secondary hemophagocytic lymphohistiocytosis in children: an analysis of etiology and outcome. J. Med. Assoc. Thai., 85: S530-541.

25. Se-Thoe, S.Y., M.M. Ng and A.E. Ling, 1999. Retrospective study of Western blot profiles in immune sera of natural dengue virus infections. J. Med. Virol., 57: 322-330.

26. Vazquez, S., M.G. Guzman, G. Guillen, G. Chinea, A.B. Perez,M. Pupo, R. Rodriguez, O. Reyes, H.E. Garay, I. Delgado, G. Garcia and M. Alvarez, 2002. Immune response to synthetic peptides of dengue prM protein. Vaccine, 20:1823-1830.
27. Cardosa, M.J., S.M. Wang, M.S. Sum and P.H. Tio, 2002. Antibodies against prM protein distinguish between previous infection with dengue and Japanese encephalitis viruses. BMC Microbiol., 2: 9.

28. Oldstone, M.B., 1998. Molecular mimicry and immune-mediated diseases. Faseb J., 12: 1255-1265.

29. Wick, G., 2000. Atherosclerosis--an autoimmune disease due to an immune reaction against heatshock protein 60. Herz., 25: 87-90.

30. Dieude, M., J.L. Senecal and Y. Raymond, 2004. Induction of endothelial cell apoptosis by heatshock protein 60-reactive antibodies from antiendothelial cell autoantibody-positive systemic lupus erythematosus patients. Arthritis Rheum., 50: 3221-3231.

31. Bason, C., R. Corrocher, C. Lunardi, P. Puccetti, O. Olivieri, D. Girelli, R. Navone, R. Beri, E. Millo, A. Margonato, N. Martinelli and A. Puccetti, 2003. Interaction of antibodies against cytomegalovirus with heat-shock protein 60 in pathogenesis of atherosclerosis. Lancet, 362: 19711977.

32. Schett, G., Q. Xu, A. Amberger, R. Van der Zee, H. Recheis, J. Willeit and G. Wick, 1995. Autoantibodies against heat shock protein 60 mediate endothelial cytotoxicity. J. Clin. Invest., 96: 2569-2577.

33. Cardosa, M.J., 1998. Dengue vaccine design: issues and challenges. Br. Med. Bull., 54: 395-405.

34. Morens, D.M., 1994. Antibody-dependent enhancement of infection and the pathogenesis of viral disease. Clin. Infect. Dis., 19: 500-512.

35. Halstead, S.B. and J. Deen, 2002. The future of dengue vaccines. Lancet, 360: 1243-1245. 